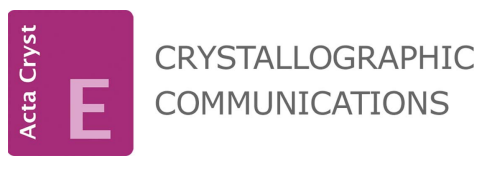

ISSN 2056-9890

Received 13 July 2018

Accepted 21 July 2018

Edited by J. Simpson, University of Otago, New Zealand

Keywords: crystal structure; iminium salt; thiazolidine ring; 2,3-dichlorobenzene; hydrogen bonding; Hirshfeld surface analysis.

CCDC reference: 1857411

Supporting information: this article has supporting information at journals.iucr.org/e

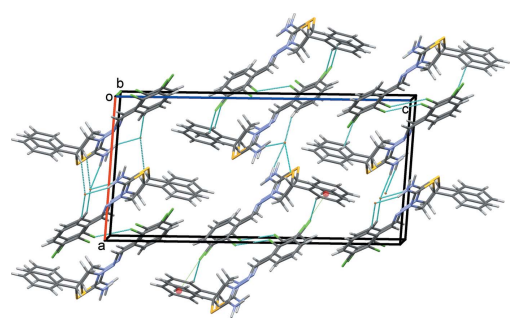

OPEN $\odot$ ACCESS

\section{Crystal structure and Hirshfeld surface analysis of (E)-3-[(2,3-dichlorobenzylidene)amino]-5-phenyl- thiazolidin-2-iminium bromide}

\author{
Mehmet Akkurt, ${ }^{a}$ Gulnara Sh. Duruskari, ${ }^{\text {b }}$ Flavien A. A. Toze, ${ }^{\text {c* }}$ Ali N. Khalilov ${ }^{\mathrm{b}}$ and \\ Afat T. Huseynova
}

\begin{abstract}
${ }^{\mathbf{a}}$ Department of Physics, Faculty of Sciences, Erciyes University, 38039 Kayseri, Turkey, ${ }^{\mathbf{b}}$ Organic Chemistry Department, Baku State University, Z. Xalilov str. 23, Az, 1148 Baku, Azerbaijan, and ${ }^{\mathbf{C}}$ Department of Chemistry, Faculty of Sciences, University of Douala, PO Box 24157, Douala, Republic of Cameroon. *Correspondence e-mail: toflavien@yahoo.fr
\end{abstract}

In the cation of the title salt, $\mathrm{C}_{16} \mathrm{H}_{14} \mathrm{Cl}_{2} \mathrm{~N}_{3} \mathrm{~S}^{+} \cdot \mathrm{Br}^{-}$, the central thiazolidine ring adopts an envelope conformation. The phenyl ring is disordered over two sites with a refined occupancy ratio of 0.541 (9):0.459 (9). In the crystal, C- $\mathrm{H} \cdots \mathrm{Br}$ and $\mathrm{N}-\mathrm{H} \cdots \mathrm{Br}$ hydrogen bonds link the components into a three-dimensional network with the cations and anions stacked along the $b$-axis direction. Weak $\mathrm{C}-\mathrm{H} \cdots \pi$ interactions, which only involve the minor disorder component of the ring, also contribute to the molecular packing. In addition, there are also inversion-related $\mathrm{Cl} \cdots \mathrm{Cl}$ halogen bonds and $\mathrm{C}-\mathrm{Cl} \cdots \pi$ (ring) contacts. A Hirshfeld surface analysis was conducted to verify the contributions of the different intermolecular interactions.

\section{Chemical context}

Schiff bases of heterocyclic amines and their complexes have attracted attention over the past decades not only due to the relatively easy synthesis, but also in view of their potential biological, pharmacological and analytical applications (Akbari et al., 2017; Gurbanov et al., 2018a,b; Hazra et al., 2018; Kvyatkovskaya et al., 2017; Mahmoudi et al., 2016, 2017a,b, 2018a,b; Mitoraj et al., 2018; Shetnev et al., 2017). Non-covalent interactions play an important role in the stabilization of coordination or supramolecular compounds derived from Schiff bases (Mahmudov et al., 2016, 2017a,b; Zubkov et al., 2018). Herein we report strong charge-assisted hydrogen bonds and halogen bonding in the structure of $(E)$ 3-[(2,3-dichlorobenzylidene)amino]-5-phenylthiazolidin-2iminium bromide.<smiles>[NH2+]=C1SC(c2ccccc2)CN1/N=C/c1cccc(Cl)c1Cl</smiles>

\section{Structural commentary}

In the cation of the title salt (Fig. 1), the central thiazolidine ring (S1/N2/C1-C3) adopts an envelope conformation with puckering parameters $Q(2)=0.205(4) \AA$ and $\varphi(2)=$ 


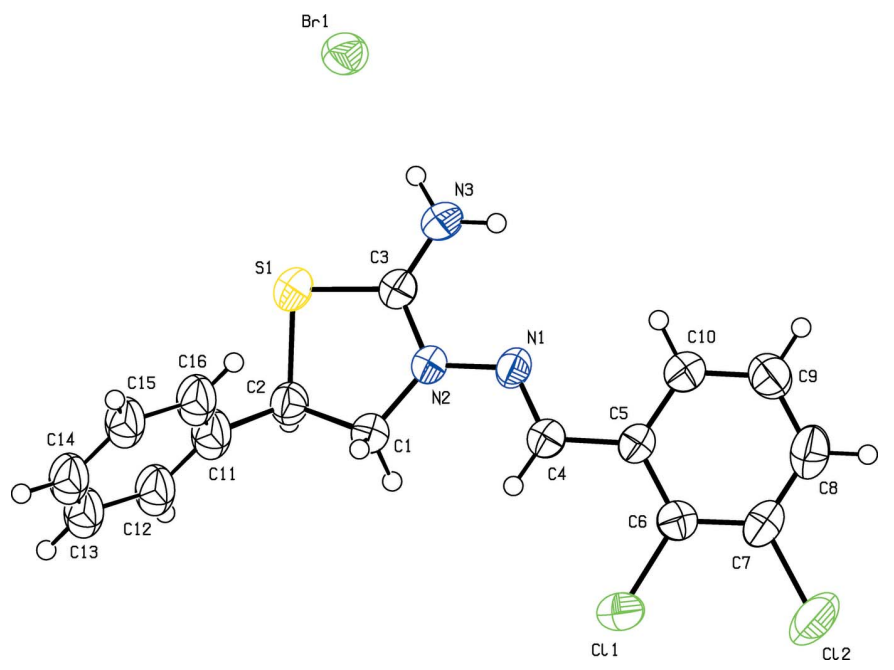

Figure 1

The molecular structure of the title salt. Displacement ellipsoids are drawn at the $50 \%$ probability level. Hydrogen atoms are shown as spheres of arbitrary radius. The minor disorder component is omitted for clarity.

$222.1(12)^{\circ}$. The dihedral angle between the mean plane of the central thiazolidine ring and the 2,3-dichlorobenzene ring $(\mathrm{C} 5-\mathrm{C} 10)$ is $16.0(2)^{\circ}$ while this plane subtends angles of 79.1 (3) and $86.7(4)^{\circ}$ with the major and minor components (C11-C16 and $\left.\mathrm{C} 11 / \mathrm{C} 12^{\prime}-\mathrm{C} 16^{\prime}\right)$, respectively, of the disordered phenyl ring. The dihedral angle between the two disorder components of the ring is $7.6(4)^{\circ}$ and these components are

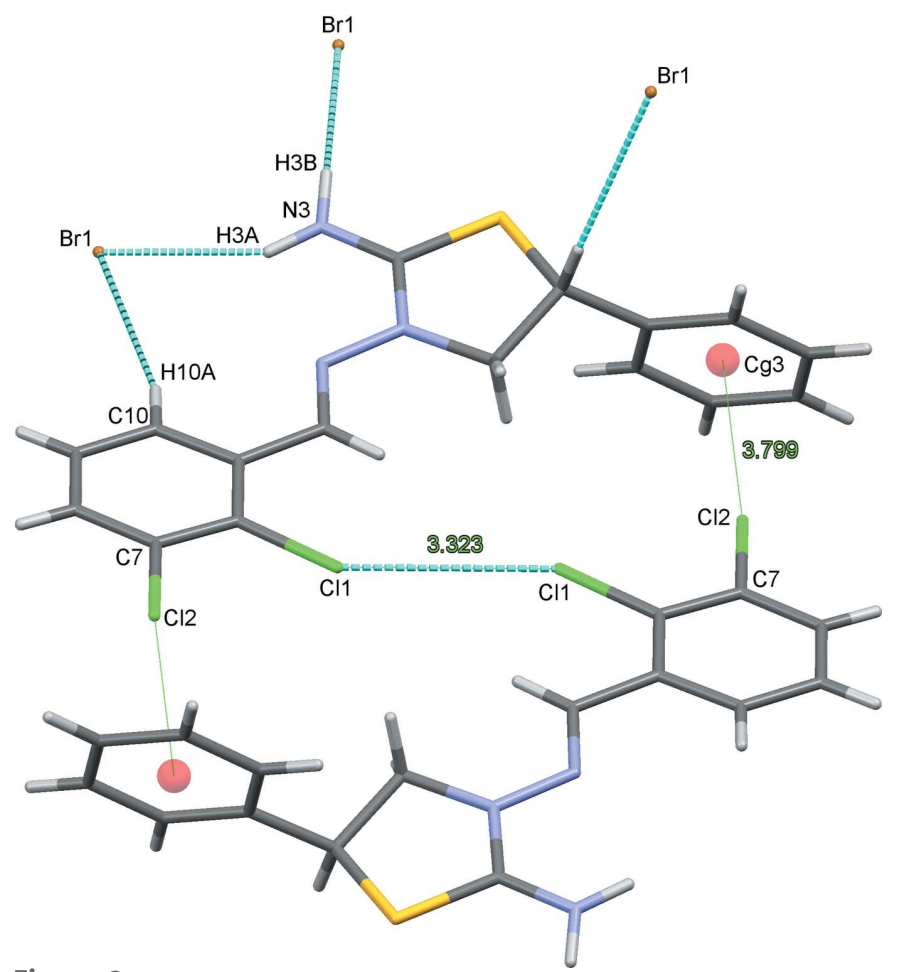

Figure 2

View of the full complement of contacts to an individual cation in the title salt. Only the major disorder component is shown. The symmetryequivalent position for the cation with the label $C g 3$ is $-x+1, y-\frac{1}{2}$, $-z+3 / 2$.
Table 1

Hydrogen-bond geometry $\left(\AA{ }^{\circ}\right)$.

$\mathrm{Cg} 3$ and $\mathrm{Cg} 4$ are the centroids of the major and minor disorder components of the $\mathrm{C} 11 / \mathrm{C} 12-\mathrm{C} 16$ and $\mathrm{C} 11 / \mathrm{C} 12^{\prime}-\mathrm{C} 16^{\prime}$ phenyl ring, respectively.

\begin{tabular}{lllll}
\hline$D-\mathrm{H} \cdots A$ & $D-\mathrm{H}$ & $\mathrm{H} \cdots A$ & $D \cdots A$ & $D-\mathrm{H} \cdots A$ \\
\hline $\mathrm{N} 3-\mathrm{H} 3 A \cdots \mathrm{Br} 1^{\mathrm{i}}$ & 0.90 & 2.51 & $3.303(4)$ & 147 \\
$\mathrm{~N} 3-\mathrm{H} 3 B \cdots \mathrm{Br} 1$ & 0.90 & 2.36 & $3.258(4)$ & 175 \\
$\mathrm{C} 13^{\prime}-\mathrm{H} 13 B \cdots C g 3^{\text {ii }}$ & 0.93 & 2.91 & $3.596(12)$ & 132 \\
$\mathrm{C} 13^{\prime}-\mathrm{H} 13 B \cdots C g 4^{\mathrm{ii}}$ & 0.93 & 2.99 & $3.746(12)$ & 139 \\
$\mathrm{C} 2-\mathrm{H} 2 A \cdots \mathrm{Br} 1^{\text {iii }}$ & 0.98 & 2.87 & $3.778(5)$ & 154 \\
$\mathrm{C} 10-\mathrm{H} 10 A \cdots \mathrm{Br} 1^{\mathrm{i}}$ & 0.93 & 2.90 & $3.796(5)$ & 161 \\
$\mathrm{C} 7-\mathrm{C} 2 \cdots C g 3^{\text {iv }}$ & $1.73(1)$ & $3.80(1)$ & $5.525(6)$ & $175(1)$ \\
$\mathrm{C} 7-\mathrm{C} 2 \cdots C g 4^{\text {iv }}$ & $1.73(1)$ & $3.57(1)$ & $5.299(6)$ & $175(1)$ \\
\hline
\end{tabular}

Symmetry codes: (i) $-x+1,-y+2,-z+1$; (ii) $-x+1, y-\frac{1}{2},-z+\frac{3}{2}$; (iii) $x, y-1, z$; (iv) $-x+2,-y,-z+1$.

oriented to the 2,3-dichlorobenzene ring by 64.8 (3) and $72.4(4)^{\circ}$, respectively. The $\mathrm{N} 2-\mathrm{N} 1-\mathrm{C} 4-\mathrm{C} 5$ bridge that links the thiazolidine and 2,3-dichlorobenzene rings has a torsion angle of $175.1(4)^{\circ}$.

\section{Supramolecular features and Hirshfeld surface analysis}

In the crystal, each cation forms $\mathrm{C}-\mathrm{H} \cdots \mathrm{Br}$ and $\mathrm{N}-\mathrm{H} \cdots \mathrm{Br}$ hydrogen bonds along with inversion-related $\mathrm{Cl} 1 \cdots \mathrm{Cl} 1$ halogen bonds and $\mathrm{C} 7-\mathrm{Cl} 2 \cdots \mathrm{Cg} 3^{\text {iv }}$ and $\mathrm{C} 7-\mathrm{Cl} 2 \cdots \mathrm{Cg} 4^{\text {iv }}$ contacts (Table 1; Fig. 2). Chains of cations form along the $a$ axis direction (Fig. 3). The crystal structure is further stabilized by $\mathrm{C} 13^{\prime}-\mathrm{H} 13 B \cdots C g 3^{\text {ii }}$ and $\mathrm{C} 13^{\prime}-\mathrm{H} 13 B \cdots C g 4^{\text {ii }}$ interactions involving the minor disorder component (Table 1). Overall, cations and anions are stacked along the $b$-axis direction (Fig. 4)

The Hirshfeld surface analysis (Spackman \& Jayatilaka, 2009) of the title salt was generated by CrystalExplorer3.1 (Wolff et al., 2012), and comprised $d_{\text {norm }}$ surface plots and two dimensional fingerprint plots (Spackman \& McKinnon, 2002). A $d_{\text {norm }}$ surface plot of the title salt is shown in Fig. 5. This plot

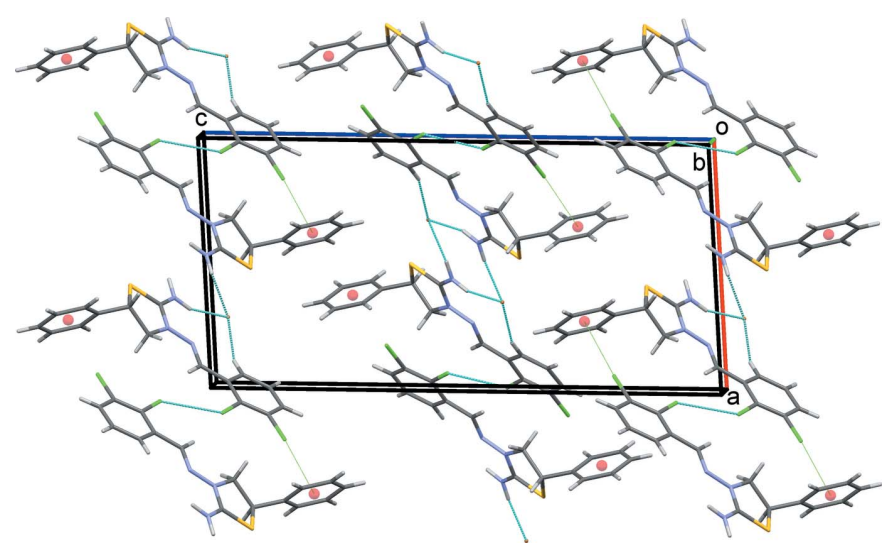

Figure 3

$\mathrm{C}-\mathrm{H} \cdots \mathrm{Br}$ and $\mathrm{N}-\mathrm{H} \cdots \mathrm{Br}$ hydrogen bonds and inversion-related $\mathrm{Cl} \cdots \mathrm{Cl}$ halogen bonds and $\mathrm{C}-\mathrm{Cl} \cdots \pi$ contacts of the title salt viewed along the $b$ axis. Only the major disorder component is shown. 


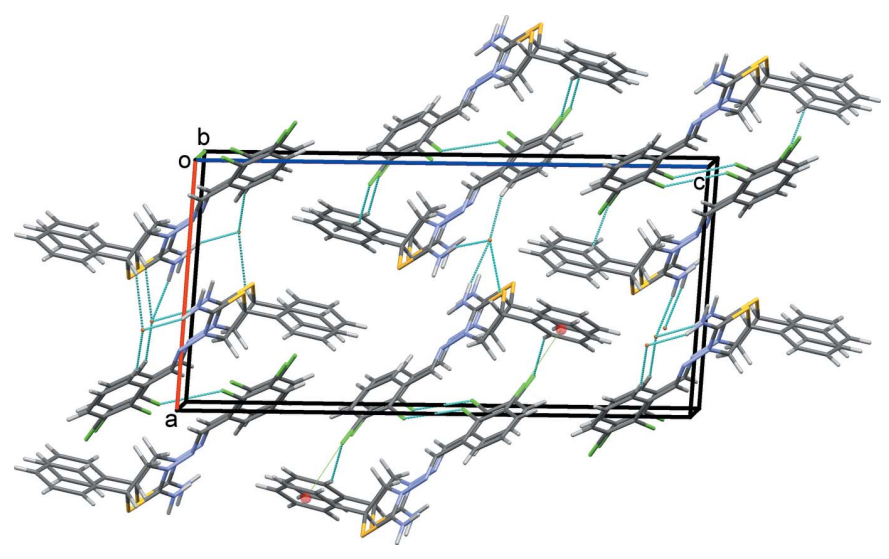

Figure 4

Overall packing of the title salt viewed along the $b$ axis. Only the major disorder component is shown.

was generated to quantify and visualize the intermolecular interactions and to explain the observed crystal packing. The dark-red spots on the $d_{\text {norm }}$ surface arise as a result of short interatomic contacts, while the other weaker intermolecular interactions appear as light-red spots.

The $d_{\text {norm }}$ surface of the title salt shows a dark-red spot at the $\mathrm{N}-\mathrm{H}$ hydrogen atom and on the bromide atom, which is the result of the strong $\mathrm{N} 3-\mathrm{H} 3 A \cdots \mathrm{Br} 1^{\mathrm{i}}$ and $\mathrm{N} 3-\mathrm{H} 3 B \cdots \mathrm{Br} 1$ hydrogen bonds present in the structure (Fig. 5). Beside these two short intermolecular contacts, the $\mathrm{C}-\mathrm{H} \cdots \mathrm{Br}$ interaction is shown as light-red spots on the $d_{\text {norm }}$ surface. The short interatomic contacts in the title salt are given in Table 2.

A quantitative analysis of the intermolecular interactions can be made by studying the fingerprint plots that are shown with characteristic pseudo-symmetry wings in the $d_{\mathrm{e}}$ and $d_{\mathrm{i}}$ diagonal axes $\left[d_{\mathrm{e}}\right.$ and $d_{\mathrm{i}}$ represent the distances from a point on the Hirshfeld surface to the nearest atoms outside (external) and inside (internal) the surface, respectively].

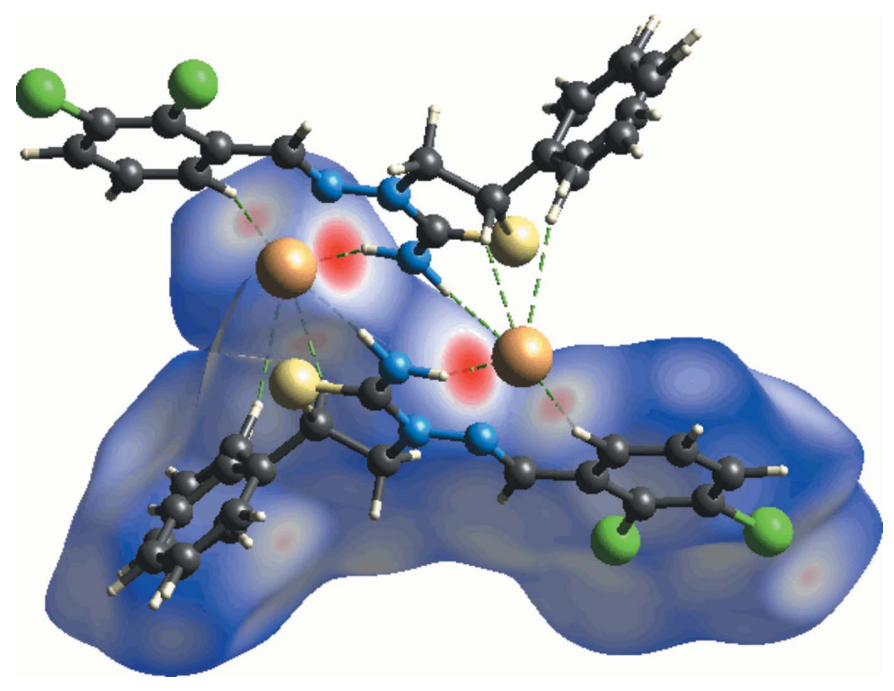

Figure 5

Hirshfeld surface of the title salt mapped with $d_{\text {norm }}$, showing the $\mathrm{C}-$ $\mathrm{H} \cdots \mathrm{Br}$ and $\mathrm{N}-\mathrm{H} \cdots \mathrm{Br}$ hydrogen bonds.
Table 3

Percentage contributions of interatomic contacts to the Hirshfeld surface for the title salt.

\begin{tabular}{ll}
\hline Contact & Percentage contribution \\
\hline $\mathrm{H} \cdots \mathrm{H}$ & 25.4 \\
$\mathrm{Cl} \cdots \mathrm{H} / \mathrm{H} \cdots \mathrm{Cl}$ & 19.1 \\
$\mathrm{C} \cdots \mathrm{H} / \mathrm{H} \cdots \mathrm{C}$ & 18.2 \\
$\mathrm{Br} \cdots \mathrm{H} / \mathrm{H} \cdots \mathrm{Br}$ & 16.2 \\
$\mathrm{~S} \cdot \mathrm{H} / \mathrm{H} \cdots \mathrm{S}$ & 5.9 \\
$\mathrm{Cl} \cdot \mathrm{C} / \mathrm{C} \cdots \mathrm{Cl}$ & 4.4 \\
$\mathrm{~N} \cdots \mathrm{H} / \mathrm{H} \cdots \mathrm{N}$ & 2.7 \\
$\mathrm{C} \cdots \mathrm{C}$ & 1.9 \\
$\mathrm{Cl} \cdot \mathrm{N} / \mathrm{N} \cdots \mathrm{Cl}$ & 1.4 \\
$\mathrm{C} \cdot \mathrm{N} / \mathrm{N} \cdots \mathrm{C}$ & 1.3 \\
$\mathrm{Br} \cdot \mathrm{C} / \mathrm{C} \cdots \mathrm{Br}$ & 1.0 \\
$\mathrm{Cl} \cdot \mathrm{Cl}$ & 0.8 \\
$\mathrm{~S} \cdots \mathrm{N} / \mathrm{N} \cdots \mathrm{S}$ & 0.7 \\
$\mathrm{~S} \cdots \mathrm{C} / \mathrm{C} \cdots \mathrm{S}$ & 0.4 \\
$\mathrm{Br} \cdot \mathrm{N} / \mathrm{N} \cdots \mathrm{Br}$ & 0.3 \\
$\mathrm{Br} \cdot \mathrm{Cl} / \mathrm{Cl} \cdots \mathrm{Br}$ & 0.3 \\
\hline
\end{tabular}

These represent both the overall two-dimensional fingerprint plots and those that represent $\mathrm{H} \cdots \mathrm{H}, \mathrm{Cl} \cdots \mathrm{H} / \mathrm{H} \cdots \mathrm{Cl}, \mathrm{C} \cdots \mathrm{H} /$ $\mathrm{H} \cdots \mathrm{C}$ and $\mathrm{Br} \cdots \mathrm{H} / \mathrm{H} \cdots \mathrm{Br}$ contacts, respectively (Fig. $6 b-e$ ). The most significant intermolecular interactions are the $\mathrm{H} \cdots \mathrm{H}$ interaction $(25.4 \%)$, which appear in the central region of the fingerprint plot with $d \mathrm{e}=d \mathrm{i} \simeq 1.2 \AA$ (Fig. 6b). The reciprocal $\mathrm{Cl} \cdots \mathrm{H} / \mathrm{H} \cdots \mathrm{Cl}$ interactions appear as two symmetrical broad wings with $d \mathrm{e}+d \mathrm{i} \simeq 2.8 \AA$ and contribute $19.1 \%$ to the Hirshfeld surface (Fig. 6c). The reciprocal C $\cdots \mathrm{H} / \mathrm{H} \cdots \mathrm{C}$ and $\mathrm{Br} \cdots \mathrm{H} / \mathrm{H} \cdots \mathrm{Br}$ interactions with $18.2 \%$ and $16.2 \%$ contributions are present as sharp symmetrical spikes at diagonal axes $d_{\mathrm{e}}+d_{\mathrm{i}} \simeq 2.7$ and $2.4 \AA$, respectively (Fig. $6 d-e$ ). The percen-
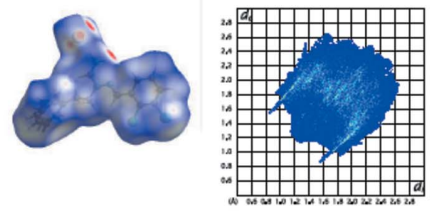

(a) All
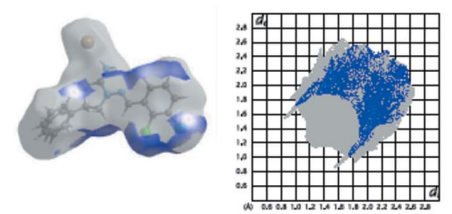

(c) $\mathrm{Cl} \cdots \mathrm{H} / \mathrm{H} \cdots \mathrm{Cl}$
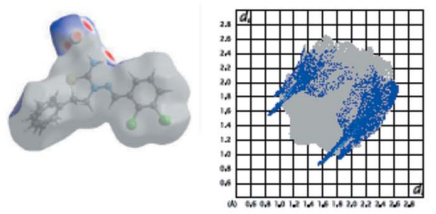

(e) $\mathrm{Br} \cdots \mathrm{H} / \mathrm{H} \cdots \mathrm{Br}$
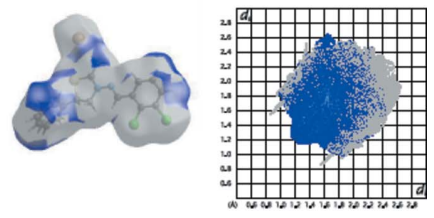

(b) $\mathrm{H} \cdots \mathrm{H}$
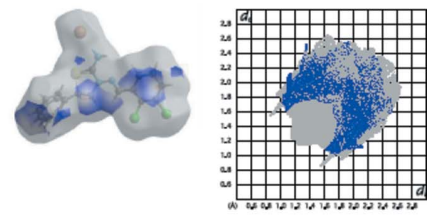

(d) $\mathrm{C} \cdots \mathrm{H} / \mathrm{H} \cdots \mathrm{C}$
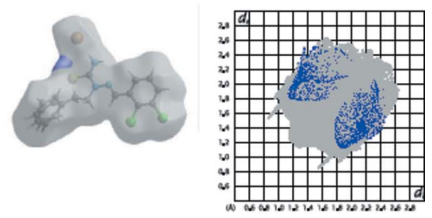

(f) $\mathrm{S} \cdots \mathrm{H} / \mathrm{H} \cdots \mathrm{S}$
Figure 6

The two-dimensional fingerprint plots of the title salt, showing (a) all interactions, and delineated into $(b) \mathrm{H} \cdots \mathrm{H},(c) \mathrm{Cl} \cdots \mathrm{H} / \mathrm{H} \cdots \mathrm{Cl},(d)$ $\mathrm{C} \cdots \mathrm{H} / \mathrm{H} \cdots \mathrm{C},(e) \mathrm{Br} \cdots \mathrm{H} / \mathrm{H} \cdots \mathrm{Br}$ and $(f) \mathrm{S} \cdots \mathrm{H} / \mathrm{H} \cdots \mathrm{S}$ interactions. 
Table 2

Summary of short interatomic contacts $(\AA)$ in the title salt.

Atoms marked with an asterisk $\left(^{*}\right)$ are from the minor component $\left(\mathrm{C} 11 / \mathrm{C} 12^{\prime}-\right.$ $\left.\mathrm{C} 16^{\prime}\right)$ of the disordered phenyl ring of the cation.

\begin{tabular}{lll}
\hline Contact & Distance & Symmetry operation \\
\hline$(\mathrm{C} 6) \mathrm{Cl} 1 \cdots \mathrm{Cl} 1(\mathrm{C} 6)$ & $3.323(2)$ & $2-x,-y, 1-z$ \\
$\left(\mathrm{C} 16^{\prime}\right) * \mathrm{H} 16 B \cdots \mathrm{H} 8 A(\mathrm{C} 8)$ & 2.56 & $2-x, 1-y, 1-z$ \\
$(\mathrm{C} 2) \mathrm{S} 1 \cdots * \mathrm{H} 14 B\left(\mathrm{C} 14^{\prime}\right)$ & 3.05 & $1-x, \frac{1}{2}+y, \frac{3}{2}-z$ \\
$(\mathrm{~N} 3) \mathrm{H} 3 B \cdots \mathrm{Br} 1$ & 2.36 & $x, y, z$ \\
$(\mathrm{~N} 3) \mathrm{H} 3 A \cdots \mathrm{Br} 1$ & 2.51 & $1-x, 2-y, 1-z$ \\
$(\mathrm{~S} 1) \mathrm{C} 3 \cdots \mathrm{C} 3(\mathrm{~S} 1)$ & $3.561(6)$ & $1-x, 1-y, 1-z$ \\
$(\mathrm{C} 1) \mathrm{H} 1 B \cdots \mathrm{Br} 1$ & 3.06 & $1-x, 1-y, 1-z$ \\
$(\mathrm{C} 5) \mathrm{C} 10 \cdots * \mathrm{H} 14 B\left(\mathrm{C} 14^{\prime}\right)$ & 2.89 & $x, \frac{1}{2}-y,-\frac{1}{2}+z$ \\
$\left(\mathrm{C} 14^{\prime}\right) * \mathrm{H} 14 B \cdots \mathrm{S} 1(\mathrm{C} 2)$ & 3.05 & $1-x,-\frac{1}{2}+y, \frac{3}{2}-z$ \\
$\left(\mathrm{C} 14^{\prime}\right) * \mathrm{H} 14 B \cdots \mathrm{C} 10(\mathrm{C} 5)$ & 2.89 & $x, \frac{1}{2}-y, \frac{1}{2}+z$ \\
$(\mathrm{C} 2) \mathrm{H} 2 A \cdots \mathrm{Br} 1$ & 2.87 & $x,-1+y, z$ \\
\hline
\end{tabular}

tage contributions of other intermolecular contacts are less than $6 \%$ in the Hirshfeld surface mapping (Table 3).

\section{Database survey}

A search of the Cambridge Structural Database (CSD Version 5.39, Nov 2017 plus three updates; Groom et al., 2016) yielded six hits for 2-thiazolidiniminium compounds with four of them reporting essentially the same cation: [WILBIC (Marthi et al., 1994), WILBOI (Marthi et al., 1994), WILBOI01 (Marthi et al., 1994), YITCEJ (Martem'yanova et al., 1993a), YITCAF (Martem'yanova et al., 1993b) and YOPLUK (Marthi et al., 1995)]. In all cases, the $3-\mathrm{N}$ atom carries a $\mathrm{C}$ substituent, not $\mathrm{N}$ as found in the title compound. The first three crystal structures were determined for racemic (WILBIC; Marthi et al., 1994) and two optically active samples (WILBOI and WILBOI01; Marthi et al., 1994) of 3-(2'-chloro-2'-phenylethyl) -2-thiazolidiniminium $p$-toluenesulfonate. In all three structures, the most disordered fragment of these molecules is the asymmetric $\mathrm{C}$ atom and the $\mathrm{Cl}$ atom attached to it. The disorder of the cation in the racemate corresponds to the presence of both enantiomers at each site in the ratio 0.821 (3): 0.179 (3). The system of hydrogen bonds connecting two cations and two anions into 12 -membered rings is identical in the racemic and in the optically active crystals. YITCEJ (Martem'yanova et al., 1993a), is a product of the interaction of 2-amino-5-methylthiazoline with methyl iodide, with alkylation at the endocylic nitrogen atom, while YITCAF (Martem'yanova et al., 1993b) is a product of the reaction of 3-nitro-5-methoxy-, 3-nitro-5-chloro-, and 3-bromo-5-nitrosalicylaldehyde with the heterocyclic base to form the salt-like complexes.

\section{Synthesis and crystallization}

To a solution of $1 \mathrm{mmol}$ of 3-amino-5-phenylthiazolidin-2iminium bromide in $20 \mathrm{~mL}$ ethanol $1 \mathrm{mmol}$ of 2,3-dichlorobenzaldehyde was added and the solution refluxed for $2 \mathrm{~h}$. The reaction mixture was cooled down to precipitate the product as colourless single crystals. These were collected by filtration
Table 4

Experimental details.

\begin{tabular}{|c|c|}
\hline \multicolumn{2}{|l|}{ Crystal data } \\
\hline Chemical formula & $\mathrm{C}_{16} \mathrm{H}_{14} \mathrm{Cl}_{2} \mathrm{~N}_{3} \mathrm{~S}^{+} \cdot \mathrm{Br}^{-}$ \\
\hline$M_{\mathrm{r}}$ & 431.17 \\
\hline Crystal system, space group & Monoclinic, $P 2_{1} / c$ \\
\hline Temperature $(\mathrm{K})$ & 296 \\
\hline$a, b, c(\AA)$ & $\begin{array}{l}11.2586(8), 6.8886(5) \\
23.0145(16)\end{array}$ \\
\hline$\beta\left(^{\circ}\right)$ & $93.678(2)$ \\
\hline$V\left(\AA^{3}\right)$ & $1781.2(2)$ \\
\hline$Z$ & 4 \\
\hline Radiation type & Мo $K \alpha$ \\
\hline$\mu\left(\mathrm{mm}^{-1}\right)$ & 2.73 \\
\hline Crystal size $(\mathrm{mm})$ & $0.28 \times 0.25 \times 0.24$ \\
\hline \multicolumn{2}{|l|}{ Data collection } \\
\hline Diffractometer & Bruker APEXII CCD \\
\hline Absorption correction & $\begin{array}{l}\text { Multi-scan (SADABS; Bruker, } \\
\text { 2007) }\end{array}$ \\
\hline$T_{\min }, T_{\max }$ & $0.483,0.546$ \\
\hline $\begin{array}{l}\text { No. of measured, independent and } \\
\text { observed }[I>2 \sigma(I)] \text { reflections }\end{array}$ & $20932,3651,2325$ \\
\hline$R_{\text {int }}$ & 0.085 \\
\hline$(\sin \theta / \lambda)_{\max }\left(\AA^{-1}\right)$ & 0.625 \\
\hline \multicolumn{2}{|l|}{ Refinement } \\
\hline$R\left[F^{2}>2 \sigma\left(F^{2}\right)\right], w R\left(F^{2}\right), S$ & $0.051,0.123,1.04$ \\
\hline No. of reflections & 3651 \\
\hline No. of parameters & 182 \\
\hline No. of restraints & 12 \\
\hline $\mathrm{H}$-atom treatment & $\mathrm{H}$-atom parameters constrained \\
\hline$\Delta \rho_{\max }, \Delta \rho_{\min }\left(\mathrm{e} \AA^{-3}\right)$ & $0.47,-0.61$ \\
\hline
\end{tabular}

Computer programs: APEX2 and SAINT (Bruker, 2007), SHELXS97 (Sheldrick, 2008), SHELXL2014 (Sheldrick, 2015), ORTEP-3 for Windows (Farrugia, 2012), Mercury (Macrae et al., 2008) and PLATON (Spek, 2003).

and washed with cold acetone. The title compound was recrystallized from methanol by slow evaporation at room temperature over several days.

Yield 89\%, m.p. 521 K. Analysis calculated for $\mathrm{C}_{16} \mathrm{H}_{14} \mathrm{BrCl}_{2} \mathrm{~N}_{3} \mathrm{~S}\left(M_{\mathrm{r}}=431.18\right): \mathrm{C}, 44.57 ; \mathrm{H}, 3.27 ; \mathrm{N}, 9.75$. Found: C, 44.51; H, 3.23; N, 9.72\%. ${ }^{1} \mathrm{H}$ NMR $(300 \mathrm{MHz}$, DMSO- $\left.d_{6}\right): 4,62\left(k, 1 \mathrm{H}, \mathrm{CH}_{2},{ }^{3} J_{\mathrm{H}-\mathrm{H}}=6.9\right) ; 4.96\left(t, 1 \mathrm{H}, \mathrm{CH}_{2}\right.$, $\left.{ }^{3} J_{\mathrm{H}-\mathrm{H}}=8.7\right) ; 5.59\left(t, 1 \mathrm{H}, \mathrm{CH}-\mathrm{Ar},{ }^{3} J_{\mathrm{H}-\mathrm{H}}=7.5\right) ; 7.38-8.50(\mathrm{~m}$, $7 \mathrm{H}, 7 \mathrm{Ar}-\mathrm{H}) ; 8.35(s, 1 \mathrm{H}, \mathrm{CH}=) ; 10.56(s, 1 \mathrm{H}, \mathrm{NH}=) .{ }^{13} \mathrm{C}$ $\operatorname{NMR}\left(75 \mathrm{MHz}, \mathrm{DMSO}-d_{6}\right)$ : 46.62, 55.68, 127.28, 127.99, 128.48, 128.96, 129.11, 132.27, 132.41, 132.51, 133.04, 137.24, 145.89, 168.92. MS (ESI), $m / z$ : $351.24\left[\mathrm{C}_{16} \mathrm{H}_{14} \mathrm{Cl}_{2} \mathrm{~N}_{3} \mathrm{~S}\right]^{+}$and $79.88 \mathrm{Br}^{-}$.

\section{Refinement}

Crystal data, data collection and structure refinement details are summarized in Table 4 . The $\mathrm{H}$ atoms were positioned geometrically $[\mathrm{N}-\mathrm{H}=0.90 \AA$ and $\mathrm{C}-\mathrm{H}=0.93-0.97 \AA]$ and were refined using a riding model, with $U_{\text {iso }}(\mathrm{H})=1.2 U_{\text {eq }}(\mathrm{C}, \mathrm{N})$. The phenyl ring in the cation is disordered over two positions with a site occupancy ratio of 0.541 (9):0.459 (9). Using DFIX, the bond distances in the two disorder components of the phenyl ring were set to $1.40 \AA$. Corresponding displacement parameters were also held to be the same using EADP. 


\section{Funding information}

This work has been partially supported by Baku State University.

\section{References}

Akbari, A. F., Mahmoudi, G., Gurbanov, A. V., Zubkov, F. I., Qu, F., Gupta, A. \& Safin, D. A. (2017). Dalton Trans. 46, 14888-14896.

Bruker (2007). APEX2, SAINT and $S A D A B S$. Bruker AXS Inc., Madison, Wisconsin, USA.

Farrugia, L. J. (2012). J. Appl. Cryst. 45, 849-854.

Groom, C. R., Bruno, I. J., Lightfoot, M. P. \& Ward, S. C. (2016). Acta Cryst. B72, 171-179.

Gurbanov, A. V., Maharramov, A. M., Zubkov, F. I., Saifutdinov, A. M. \& Guseinov, F. I. (2018a). Aust. J. Chem. 71, 190-194.

Gurbanov, A. V., Mahmoudi, G., Guedes da Silva, M. F. C., Zubkov, F. I., Mahmudov, K. T. \& Pombeiro, A. J. L. (2018b). Inorg. Chim. Acta, 471, 130-136.

Hazra, S., Martins, N. M. R., Mahmudov, K. T., Zubkov, F. I., Guedes da Silva, M. F. C. \& Pombeiro, A. J. L. (2018). J. Organomet. Chem. 867, 197-200.

Kvyatkovskaya, E. A., Zaytsev, V. P., Zubkov, F. I., Dorovatovskii, P. V., Zubavichus, Y. V. \& Khrustalev, V. N. (2017). Acta Cryst. E73, 515-519.

Macrae, C. F., Bruno, I. J., Chisholm, J. A., Edgington, P. R., McCabe, P., Pidcock, E., Rodriguez-Monge, L., Taylor, R., van de Streek, J. \& Wood, P. A. (2008). J. Appl. Cryst. 41, 466-470.

Mahmoudi, G., Afkhami, F. A., Castiñeiras, A., García-Santos, I., Gurbanov, A., Zubkov, F. I., Mitoraj, M. P., Kukułka, M., Sagan, F., Szczepanik, D. W., Konyaeva, I. A. \& Safin, D. A. (2018). Inorg. Chem. 57, 4395-4408.

Mahmoudi, G., Bauzá, A., Gurbanov, A. V., Zubkov, F. I., Maniukiewicz, W., Rodríguez-Diéguez, A., López-Torres, E. \& Frontera, A. (2016). CrystEngComm, 18, 9056-9066.

Mahmoudi, G., Gurbanov, A. V., Rodríguez-Hermida, S., Carballo, R., Amini, M., Bacchi, A., Mitoraj, M. P., Sagan, F., Kukułka, M. \& Safin, D. A. (2017a). Inorg. Chem. 56, 9698-9709.
Mahmoudi, G., Seth, S. K., Bauzá, A., Zubkov, F. I., Gurbanov, A. V., White, J., Stilinović, V., Doert, Th. \& Frontera, A. (2018a). CrystEngComm, 20, 2812-2821.

Mahmoudi, G., Zangrando, E., Bauzá, A., Maniukiewicz, W., Carballo, R., Gurbanov, A. V. \& Frontera, A. (2017b). CrystEngComm, 19, 3322-3330.

Mahmoudi, G., Zaręba, J. K., Gurbanov, A. V., Bauzá, A., Zubkov, F. I., Kubicki, M., Stilinović, V., Kinzhybalo, V. \& Frontera, A. (2018b). Eur. J. Inorg. Chem. 4763-4772.

Mahmudov, K. T., Kopylovich, M. N., Guedes da Silva, M. F. C. \& Pombeiro, A. J. L. (2017a). Coord. Chem. Rev. 345, 54-72.

Mahmudov, K. T., Kopylovich, M. N., Guedes da Silva, M. F. C. \& Pombeiro, A. J. L. (2017b). Dalton Trans. 46, 10121-10138.

Mahmudov, K. T. \& Pombeiro, A. J. L. (2016). Chem. Eur. J. 22, 16356-16398.

Martem'yanova, N. A., Chunaev, Y. M., Przhiyalgovskaya, N. M., Kurkovskaya, L. N., Filipenko, O. S. \& Aldoshin, S. M. (1993a). Khim. Geterotsikl. Soedin. pp. 415-419.

Martem'yanova, N. A., Chunaev, Y. M., Przhiyalgovskaya, N. M., Kurkovskaya, L. N., Filipenko, O. S. \& Aldoshin, S. M. (1993b). Khim. Geterotsikl. Soedin. pp. 420-425.

Marthi, K., Larsen, S., Ács, M., Bálint, J. \& Fogassy, E. (1994). Acta Cryst. B50, 762-771.

Marthi, K., Larsen, M., Ács, M., Bálint, J. \& Fogassy, E. (1995). Acta Chem. Scand. 49, 20-27.

Sheldrick, G. M. (2008). Acta Cryst. A64, 112-122.

Sheldrick, G. M. (2015). Acta Cryst. C71, 3-8.

Shetnev, A. A. \& Zubkov, F. I. (2017). Chem. Heterocycl. C. 53, 495497.

Spackman, M. \& Jayatilaka, D. (2009). CrystEngComm, 11, 19-32.

Spackman, M. A. \& McKinnon, J. J. (2002). CrystEngComm, 4, 378392.

Spek, A. L. (2003). J. Appl. Cryst. 36, 7-13.

Wolff, S. K., Grimwood, D. J., McKinnon, J. J., Turner, M. J., Jayatilaka, D. \& Spackman, M. A. (2012). Crystal Explorer. University of Western Australia.

Zubkov, F. I., Mertsalov, D. F., Zaytsev, V. P., Varlamov, A. V., Gurbanov, A. V., Dorovatovskii, P. V., Timofeeva, T. V., Khrustalev, V. N. \& Mahmudov, K. T. (2018). J. Mol. Liq. 249, 949-952. 


\section{supporting information}

Acta Cryst. (2018). E74, 1168-1172［https://doi.org/10.1107/S2056989018010496]

Crystal structure and Hirshfeld surface analysis of (E)-3-[(2,3-dichlorobenzylidene)amino]-5-phenylthiazolidin-2-iminium bromide

Mehmet Akkurt, Gulnara Sh. Duruskari, Flavien A. A. Toze, Ali N. Khalilov and Afat T.

Huseynova

Computing details

Data collection: APEX2 (Bruker, 2007); cell refinement: SAINT (Bruker, 2007); data reduction: SAINT (Bruker, 2007); program(s) used to solve structure: SHELXS97 (Sheldrick, 2008); program(s) used to refine structure: SHELXL2014 (Sheldrick, 2015); molecular graphics: ORTEP-3 for Windows (Farrugia, 2012) and Mercury (Macrae et al., 2008); software used to prepare material for publication: PLATON (Spek, 2003).

(E)-3-[(2,3-Dichlorobenzylidene)amino]-5-phenylthiazolidin-2-iminium bromide

Crystal data

$\mathrm{C}_{16} \mathrm{H}_{14} \mathrm{Cl}_{2} \mathrm{~N}_{3} \mathrm{~S}^{+} \cdot \mathrm{Br}^{-}$

$M_{r}=431.17$

Monoclinic, $P 2_{1} / c$

$a=11.2586(8) \AA$

$b=6.8886(5) \AA$

$c=23.0145(16) \AA$

$\beta=93.678(2)^{\circ}$

$V=1781.2(2) \AA^{3}$

$Z=4$

\section{Data collection}

Bruker APEXII CCD diffractometer

$\varphi$ and $\omega$ scans

Absorption correction: multi-scan

(SADABS; Bruker, 2007)

$T_{\min }=0.483, T_{\max }=0.546$

20932 measured reflections

\section{Refinement}

Refinement on $F^{2}$

Least-squares matrix: full

$R\left[F^{2}>2 \sigma\left(F^{2}\right)\right]=0.051$

$w R\left(F^{2}\right)=0.123$

$S=1.04$

3651 reflections

182 parameters

12 restraints
$F(000)=864$

$D_{\mathrm{x}}=1.608 \mathrm{Mg} \mathrm{m}^{-3}$

Mo $K \alpha$ radiation, $\lambda=0.71073 \AA$

Cell parameters from 5051 reflections

$\theta=2.5-24.3^{\circ}$

$\mu=2.73 \mathrm{~mm}^{-1}$

$T=296 \mathrm{~K}$

Block, colourless

$0.28 \times 0.25 \times 0.24 \mathrm{~mm}$

3651 independent reflections

2325 reflections with $I>2 \sigma(I)$

$R_{\text {int }}=0.085$

$\theta_{\max }=26.4^{\circ}, \theta_{\min }=2.5^{\circ}$

$h=-14 \rightarrow 14$

$k=-8 \rightarrow 8$

$l=-28 \rightarrow 28$

Primary atom site location: structure-invariant direct methods

Secondary atom site location: difference Fourier map

Hydrogen site location: mixed

$\mathrm{H}$-atom parameters constrained

$w=1 /\left[\sigma^{2}\left(F_{\mathrm{o}}^{2}\right)+(0.0342 P)^{2}+3.7192 P\right]$

where $P=\left(F_{\mathrm{o}}{ }^{2}+2 F_{\mathrm{c}}{ }^{2}\right) / 3$ 
$(\Delta / \sigma)_{\max }=0.001$

$\Delta \rho_{\min }=-0.61$ e $\AA^{-3}$

$\Delta \rho_{\max }=0.47$ e $\AA^{-3}$

Special details

Geometry. All esds (except the esd in the dihedral angle between two 1.s. planes) are estimated using the full covariance matrix. The cell esds are taken into account individually in the estimation of esds in distances, angles and torsion angles; correlations between esds in cell parameters are only used when they are defined by crystal symmetry. An approximate (isotropic) treatment of cell esds is used for estimating esds involving l.s. planes.

Fractional atomic coordinates and isotropic or equivalent isotropic displacement parameters $\left(\hat{A}^{2}\right)$

\begin{tabular}{|c|c|c|c|c|c|}
\hline & $x$ & $y$ & $z$ & $U_{\text {iso }} * / U_{\text {eq }}$ & Occ. $(<1)$ \\
\hline $\mathrm{Br} 1$ & $0.33815(4)$ & $0.95208(8)$ & $0.56902(2)$ & 0.05678 (19) & \\
\hline $\mathrm{Cl1}$ & $1.02426(13)$ & $0.1283(2)$ & $0.44079(7)$ & $0.0687(4)$ & \\
\hline $\mathrm{Cl} 2$ & $1.12966(15)$ & $0.2307(2)$ & $0.32291(7)$ & $0.0869(5)$ & \\
\hline $\mathrm{S} 1$ & $0.52713(12)$ & $0.4896(2)$ & $0.61266(6)$ & $0.0624(4)$ & \\
\hline N1 & $0.7591(3)$ & $0.5219(5)$ & $0.49730(15)$ & $0.0408(9)$ & \\
\hline N2 & $0.6952(3)$ & $0.4605(6)$ & $0.54294(15)$ & $0.0433(9)$ & \\
\hline N3 & $0.5794(4)$ & $0.7393(6)$ & $0.53057(18)$ & $0.0579(12)$ & \\
\hline $\mathrm{H} 3 \mathrm{~A}$ & 0.624225 & 0.782615 & 0.502288 & $0.069 *$ & \\
\hline H3B & 0.510005 & 0.793065 & 0.539518 & $0.069 *$ & \\
\hline $\mathrm{C} 1$ & $0.7176(4)$ & $0.2900(7)$ & $0.5795(2)$ & $0.0465(11)$ & \\
\hline $\mathrm{H} 1 \mathrm{~A}$ & 0.787141 & 0.310790 & 0.605841 & $0.056^{*}$ & \\
\hline H1B & 0.731818 & 0.177512 & 0.555530 & $0.056 *$ & \\
\hline $\mathrm{C} 2$ & $0.6072(4)$ & $0.2576(7)$ & $0.6139(2)$ & $0.0456(11)$ & \\
\hline $\mathrm{H} 2 \mathrm{~A}$ & 0.556596 & 0.159930 & 0.593684 & $0.055^{*}$ & \\
\hline $\mathrm{C} 3$ & $0.6050(4)$ & $0.5758(7)$ & $0.5566(2)$ & $0.0436(11)$ & \\
\hline $\mathrm{C} 4$ & $0.8437(4)$ & $0.4154(6)$ & $0.48214(18)$ & $0.0380(10)$ & \\
\hline $\mathrm{H} 4 \mathrm{~A}$ & 0.865728 & 0.304542 & 0.503231 & $0.046^{*}$ & \\
\hline $\mathrm{C} 5$ & $0.9060(4)$ & $0.4708(6)$ & $0.43080(18)$ & $0.0368(10)$ & \\
\hline C6 & $0.9857(4)$ & $0.3433(6)$ & 0.40648 (19) & $0.0414(10)$ & \\
\hline $\mathrm{C} 7$ & $1.0339(4)$ & $0.3904(8)$ & $0.3543(2)$ & $0.0514(13)$ & \\
\hline $\mathrm{C} 8$ & $1.0074(4)$ & $0.5637(9)$ & $0.3272(2)$ & $0.0608(14)$ & \\
\hline H8A & 1.039350 & 0.593679 & 0.292068 & $0.073^{*}$ & \\
\hline C9 & $0.9330(5)$ & $0.6929(8)$ & $0.3525(2)$ & $0.0586(14)$ & \\
\hline H9A & 0.916921 & 0.812359 & 0.334891 & $0.070^{*}$ & \\
\hline $\mathrm{C} 10$ & $0.8825(4)$ & $0.6470(7)$ & $0.4033(2)$ & 0.0487 (12) & \\
\hline H10A & 0.831686 & 0.735272 & 0.419627 & $0.058 *$ & \\
\hline C11 & $0.6345(4)$ & $0.1918(6)$ & $0.67564(14)$ & $0.0609(8)$ & \\
\hline $\mathrm{C} 12$ & $0.6080(6)$ & $0.0096(6)$ & $0.6973(3)$ & $0.0609(8)$ & $0.541(9)$ \\
\hline $\mathrm{H} 12 \mathrm{~A}$ & 0.571907 & -0.083422 & 0.672760 & $0.073 *$ & $0.541(9)$ \\
\hline C13 & $0.6355(8)$ & $-0.0337(10)$ & $0.7556(3)$ & $0.0609(8)$ & $0.541(9)$ \\
\hline $\mathrm{H} 13 \mathrm{~A}$ & 0.617773 & -0.155583 & 0.770084 & $0.073 *$ & $0.541(9)$ \\
\hline C14 & $0.6895(7)$ & $0.1053(15)$ & $0.79226(18)$ & $0.0609(8)$ & $0.541(9)$ \\
\hline H14A & 0.707872 & 0.076397 & 0.831271 & $0.073^{*}$ & $0.541(9)$ \\
\hline C15 & $0.7160(6)$ & $0.2875(13)$ & $0.7706(2)$ & $0.0609(8)$ & $0.541(9)$ \\
\hline $\mathrm{H} 15 \mathrm{~A}$ & 0.752105 & 0.380540 & 0.795135 & $0.073 *$ & $0.541(9)$ \\
\hline C16 & $0.6885(6)$ & $0.3308(7)$ & $0.7123(2)$ & $0.0609(8)$ & $0.541(9)$ \\
\hline
\end{tabular}




\begin{tabular}{llllll} 
H16A & 0.706240 & 0.452706 & 0.697811 & $0.073^{*}$ & $0.541(9)$ \\
C12' & $0.5874(10)$ & $0.0071(10)$ & $0.6850(4)$ & $0.0609(8)$ & $0.459(9)$ \\
H12B & 0.540937 & -0.050445 & 0.654817 & $0.073^{*}$ & $0.459(9)$ \\
C13' & $0.6064(10)$ & $-0.0955(17)$ & $0.7373(4)$ & $0.0609(8)$ & $0.459(9)$ \\
H13B & 0.575083 & -0.219058 & 0.741912 & $0.073^{*}$ & $0.459(9)$ \\
C14' & $0.6746(10)$ & $-0.0036(19)$ & $0.7822(5)$ & $0.0609(8)$ & $0.459(9)$ \\
H14B & 0.690965 & -0.069494 & 0.817074 & $0.073^{*}$ & $0.459(9)$ \\
C15' & $0.7188(10)$ & $0.1846(18)$ & $0.7762(4)$ & $0.0609(8)$ & $0.459(9)$ \\
H15B & 0.758883 & 0.247056 & 0.807429 & $0.073^{*}$ & $0.459(9)$ \\
C16' & $0.7016(9)$ & $0.2771(16)$ & $0.7221(3)$ & $0.0609(8)$ & $0.459(9)$ \\
H16B & 0.735675 & 0.398420 & 0.716985 & $0.073^{*}$ & $0.459(9)$ \\
\hline
\end{tabular}

Atomic displacement parameters $\left(\AA^{2}\right)$

\begin{tabular}{|c|c|c|c|c|c|c|}
\hline & $U^{11}$ & $U^{22}$ & $U^{33}$ & $U^{12}$ & $U^{13}$ & $U^{23}$ \\
\hline Brl & 0.0494 & 0.0517 (3) & $0.0702(4)$ & $0.0042(3)$ & $0.0114(2)$ & 0.0059 (3) \\
\hline $\mathrm{Cl1}$ & $0.0778(9)$ & $0.0476(8)$ & $0.0840(10)$ & $0.0186(7)$ & $0.0307(8)$ & $0.0078(7)$ \\
\hline $\mathrm{Cl} 2$ & $0.0899(11)$ & $0.0856(11)$ & $0.0910(12)$ & $0.0098(9)$ & $0.0511(9)$ & $-0.0178(9)$ \\
\hline $\mathrm{S} 1$ & $0.0564(8)$ & $0.0674(9)$ & $0.0669(9)$ & $0.0151(7)$ & $0.0308(7)$ & $0.0139(7)$ \\
\hline $\mathrm{N} 1$ & $0.041(2)$ & $0.044(2)$ & $0.039(2)$ & $-0.0015(17)$ & $0.0120(16)$ & $-0.0025(17)$ \\
\hline $\mathrm{N} 2$ & $0.045(2)$ & $0.046(2)$ & $0.041(2)$ & $0.0077(18)$ & $0.0128(17)$ & $0.0048(18)$ \\
\hline N3 & $0.052(2)$ & $0.057(3)$ & $0.067(3)$ & $0.019(2)$ & $0.025(2)$ & $0.011(2)$ \\
\hline $\mathrm{C} 1$ & 0.049 (3) & 0.049 & $0.042(3)$ & $0.007(2)$ & 0.009 (2) & $0.004(2)$ \\
\hline $\mathrm{C} 2$ & $0.043(3)$ & $0.051(3)$ & $0.044(3)$ & $-0.001(2)$ & $0.008(2)$ & $0.003(2)$ \\
\hline $\mathrm{C} 3$ & $0.042(2)$ & 0.045 & $0.044(3)$ & $0.002(2)$ & 0.009 (2) & $0.001(2)$ \\
\hline $\mathrm{C} 4$ & $0.038(2)$ & $0.039(3)$ & $0.037(2)$ & $-0.0026(19)$ & $0.0010(19)$ & $-0.0036(19)$ \\
\hline $\mathrm{C} 5$ & $0.033(2)$ & $0.040(2)$ & $0.037(2)$ & $-0.0042(19)$ & $0.0035(18)$ & $-0.005(2)$ \\
\hline C6 & $0.039(2)$ & 0.039 & $0.046(3)$ & $-0.004(2)$ & $0.002(2)$ & $-0.002(2)$ \\
\hline $\mathrm{C} 7$ & $0.048(3)$ & 0.059 (3) & $0.049(3)$ & $-0.004(2)$ & $0.015(2)$ & $-0.012(3)$ \\
\hline $\mathrm{C} 8$ & $0.051(3)$ & $0.084(4)$ & $0.048(3)$ & $-0.004(3)$ & $0.012(2)$ & 0.004 (3) \\
\hline C9 & 0.055 & $0.061(3)$ & 0.060 & $0.005(3)$ & 0.007 (3) & 0.015 \\
\hline $\mathrm{C} 10$ & $0.047(3)$ & $0.050(3)$ & $0.049(3)$ & $0.006(2)$ & $0.008(2)$ & $0.000(2)$ \\
\hline $\mathrm{C} 11$ & $0.0495(16)$ & $0.090(2)$ & $0.0436(16)$ & $0.0106(16)$ & $0.0043(12)$ & $0.0150(15)$ \\
\hline $\mathrm{C} 12$ & $0.0495(16)$ & $0.090(2)$ & $0.0436(16)$ & $0.0106(16)$ & $0.0043(12)$ & $0.0150(15)$ \\
\hline $\mathrm{C} 13$ & $0.0495(16)$ & $0.090(2)$ & $0.0436(16)$ & $0.0106(16)$ & $0.0043(12)$ & $0.0150(15)$ \\
\hline C14 & $0.0495(16)$ & $0.090(2)$ & $0.0436(16)$ & $0.0106(16)$ & $0.0043(12)$ & $0.0150(15)$ \\
\hline $\mathrm{C} 15$ & $0.0495(16)$ & $0.090(2)$ & $0.0436(16)$ & $0.0106(16)$ & $0.0043(12)$ & $0.0150(15)$ \\
\hline $\mathrm{C} 16$ & $0.0495(16)$ & $0.090(2)$ & $0.0436(16)$ & $0.0106(16)$ & $0.0043(12)$ & $0.0150(15)$ \\
\hline C12' & $0.0495(16)$ & $0.090(2)$ & $0.0436(16)$ & $0.0106(16)$ & $0.0043(12)$ & $0.0150(15)$ \\
\hline C13' & $0.0495(16)$ & $0.090(2)$ & $0.0436(16)$ & $0.0106(16)$ & $0.0043(12)$ & $0.0150(15)$ \\
\hline C14' & $0.0495(16)$ & $0.090(2)$ & $0.0436(16)$ & $0.0106(16)$ & $0.0043(12)$ & $0.0150(15)$ \\
\hline $\mathrm{C} 15^{\prime}$ & $0.0495(16)$ & $0.090(2)$ & $0.0436(16)$ & $0.0106(16)$ & $0.0043(12)$ & $0.0150(15)$ \\
\hline $\mathrm{C} 16^{\prime}$ & $0.0495(16)$ & $0.090(2)$ & $0.0436(16)$ & $0.0106(16)$ & $0.0043(12)$ & $0.0150(15)$ \\
\hline
\end{tabular}

Geometric parameters $\left(\AA,{ }^{\circ}\right)$

\begin{tabular}{llll}
\hline $\mathrm{C} 11-\mathrm{C} 6$ & $1.720(5)$ & $\mathrm{C} 9-\mathrm{H} 9 \mathrm{~A}$ & 0.9300 \\
$\mathrm{Cl} 2-\mathrm{C} 7$ & $1.730(5)$ & $\mathrm{C} 10-\mathrm{H} 10 \mathrm{~A}$ & 0.9300
\end{tabular}




\begin{tabular}{|c|c|c|c|}
\hline $\mathrm{S} 1-\mathrm{C} 3$ & $1.712(5)$ & $\mathrm{C} 11-\mathrm{C} 12$ & 1.3900 \\
\hline $\mathrm{S} 1-\mathrm{C} 2$ & $1.834(5)$ & $\mathrm{C} 11-\mathrm{C} 16$ & 1.3900 \\
\hline $\mathrm{N} 1-\mathrm{C} 4$ & $1.269(5)$ & $\mathrm{C} 11-\mathrm{C} 16^{\prime}$ & $1.399(2)$ \\
\hline $\mathrm{N} 1-\mathrm{N} 2$ & $1.377(5)$ & $\mathrm{C} 11-\mathrm{C} 12^{\prime}$ & $1.400(2)$ \\
\hline $\mathrm{N} 2-\mathrm{C} 3$ & $1.342(5)$ & $\mathrm{C} 12-\mathrm{C} 13$ & 1.3900 \\
\hline $\mathrm{N} 2-\mathrm{C} 1$ & $1.457(6)$ & $\mathrm{C} 12-\mathrm{H} 12 \mathrm{~A}$ & 0.9300 \\
\hline $\mathrm{N} 3-\mathrm{C} 3$ & $1.299(6)$ & $\mathrm{C} 13-\mathrm{C} 14$ & 1.3900 \\
\hline N3-H3A & 0.9000 & $\mathrm{C} 13-\mathrm{H} 13 \mathrm{~A}$ & 0.9300 \\
\hline N3-H3B & 0.9001 & $\mathrm{C} 14-\mathrm{C} 15$ & 1.3900 \\
\hline $\mathrm{C} 1-\mathrm{C} 2$ & $1.533(6)$ & $\mathrm{C} 14-\mathrm{H} 14 \mathrm{~A}$ & 0.9300 \\
\hline $\mathrm{C} 1-\mathrm{H} 1 \mathrm{~A}$ & 0.9700 & $\mathrm{C} 15-\mathrm{C} 16$ & 1.3900 \\
\hline $\mathrm{C} 1-\mathrm{H} 1 \mathrm{~B}$ & 0.9700 & C15-H15A & 0.9300 \\
\hline $\mathrm{C} 2-\mathrm{C} 11$ & $1.503(6)$ & C16-H16A & 0.9300 \\
\hline $\mathrm{C} 2-\mathrm{H} 2 \mathrm{~A}$ & 0.9800 & $\mathrm{C} 12^{\prime}-\mathrm{C} 13^{\prime}$ & $1.400(2)$ \\
\hline $\mathrm{C} 4-\mathrm{C} 5$ & $1.463(6)$ & $\mathrm{C} 12^{\prime}-\mathrm{H} 12 \mathrm{~B}$ & 0.9300 \\
\hline $\mathrm{C} 4-\mathrm{H} 4 \mathrm{~A}$ & 0.9300 & $\mathrm{C} 13^{\prime}-\mathrm{C} 14^{\prime}$ & $1.400(2)$ \\
\hline $\mathrm{C} 5-\mathrm{C} 10$ & $1.386(6)$ & $\mathrm{C} 13^{\prime}-\mathrm{H} 13 \mathrm{~B}$ & 0.9300 \\
\hline $\mathrm{C} 5-\mathrm{C} 6$ & $1.398(6)$ & $\mathrm{C} 14^{\prime}-\mathrm{C} 15^{\prime}$ & $1.399(2)$ \\
\hline $\mathrm{C} 6-\mathrm{C} 7$ & $1.388(6)$ & C14'-H14B & 0.9300 \\
\hline $\mathrm{C} 7-\mathrm{C} 8$ & $1.370(7)$ & $\mathrm{C} 15^{\prime}-\mathrm{C} 16^{\prime}$ & $1.400(2)$ \\
\hline $\mathrm{C} 8-\mathrm{C} 9$ & $1.376(7)$ & $\mathrm{C} 15^{\prime}-\mathrm{H} 15 \mathrm{~B}$ & 0.9300 \\
\hline $\mathrm{C} 8-\mathrm{H} 8 \mathrm{~A}$ & 0.9300 & $\mathrm{C} 16^{\prime}-\mathrm{H} 16 \mathrm{~B}$ & 0.9300 \\
\hline $\mathrm{C} 9-\mathrm{C} 10$ & $1.371(7)$ & & \\
\hline $\mathrm{C} 3-\mathrm{S} 1-\mathrm{C} 2$ & $92.3(2)$ & $\mathrm{C} 8-\mathrm{C} 9-\mathrm{H} 9 \mathrm{~A}$ & 119.7 \\
\hline $\mathrm{C} 4-\mathrm{N} 1-\mathrm{N} 2$ & $118.1(4)$ & $\mathrm{C} 9-\mathrm{C} 10-\mathrm{C} 5$ & $120.9(5)$ \\
\hline $\mathrm{C} 3-\mathrm{N} 2-\mathrm{N} 1$ & $115.9(4)$ & $\mathrm{C} 9-\mathrm{C} 10-\mathrm{H} 10 \mathrm{~A}$ & 119.5 \\
\hline $\mathrm{C} 3-\mathrm{N} 2-\mathrm{C} 1$ & $116.6(4)$ & $\mathrm{C} 5-\mathrm{C} 10-\mathrm{H} 10 \mathrm{~A}$ & 119.5 \\
\hline $\mathrm{N} 1-\mathrm{N} 2-\mathrm{C} 1$ & $127.4(3)$ & $\mathrm{C} 12-\mathrm{C} 11-\mathrm{C} 16$ & 120.0 \\
\hline $\mathrm{C} 3-\mathrm{N} 3-\mathrm{H} 3 \mathrm{~A}$ & 120.2 & $\mathrm{C} 16^{\prime}-\mathrm{C} 11-\mathrm{C} 12^{\prime}$ & $117.1(6)$ \\
\hline $\mathrm{C} 3-\mathrm{N} 3-\mathrm{H} 3 \mathrm{~B}$ & 114.9 & $\mathrm{C} 12-\mathrm{C} 11-\mathrm{C} 2$ & $125.1(4)$ \\
\hline $\mathrm{H} 3 \mathrm{~A}-\mathrm{N} 3-\mathrm{H} 3 \mathrm{~B}$ & 124.4 & $\mathrm{C} 16-\mathrm{C} 11-\mathrm{C} 2$ & $114.9(4)$ \\
\hline $\mathrm{N} 2-\mathrm{C} 1-\mathrm{C} 2$ & $107.4(4)$ & $\mathrm{C} 16^{\prime}-\mathrm{C} 11-\mathrm{C} 2$ & $131.6(6)$ \\
\hline $\mathrm{N} 2-\mathrm{C} 1-\mathrm{H} 1 \mathrm{~A}$ & 110.2 & $\mathrm{C} 12^{\prime}-\mathrm{C} 11-\mathrm{C} 2$ & $111.2(5)$ \\
\hline $\mathrm{C} 2-\mathrm{C} 1-\mathrm{H} 1 \mathrm{~A}$ & 110.2 & $\mathrm{C} 13-\mathrm{C} 12-\mathrm{C} 11$ & 120.0 \\
\hline $\mathrm{N} 2-\mathrm{C} 1-\mathrm{H} 1 \mathrm{~B}$ & 110.2 & $\mathrm{C} 13-\mathrm{C} 12-\mathrm{H} 12 \mathrm{~A}$ & 120.0 \\
\hline $\mathrm{C} 2-\mathrm{C} 1-\mathrm{H} 1 \mathrm{~B}$ & 110.2 & $\mathrm{C} 11-\mathrm{C} 12-\mathrm{H} 12 \mathrm{~A}$ & 120.0 \\
\hline $\mathrm{H} 1 \mathrm{~A}-\mathrm{C} 1-\mathrm{H} 1 \mathrm{~B}$ & 108.5 & $\mathrm{C} 12-\mathrm{C} 13-\mathrm{C} 14$ & 120.0 \\
\hline $\mathrm{C} 11-\mathrm{C} 2-\mathrm{C} 1$ & $114.2(4)$ & $\mathrm{C} 12-\mathrm{C} 13-\mathrm{H} 13 \mathrm{~A}$ & 120.0 \\
\hline $\mathrm{C} 11-\mathrm{C} 2-\mathrm{S} 1$ & $110.4(3)$ & $\mathrm{C} 14-\mathrm{C} 13-\mathrm{H} 13 \mathrm{~A}$ & 120.0 \\
\hline $\mathrm{C} 1-\mathrm{C} 2-\mathrm{S} 1$ & $106.2(3)$ & $\mathrm{C} 13-\mathrm{C} 14-\mathrm{C} 15$ & 120.0 \\
\hline $\mathrm{C} 11-\mathrm{C} 2-\mathrm{H} 2 \mathrm{~A}$ & 108.7 & $\mathrm{C} 13-\mathrm{C} 14-\mathrm{H} 14 \mathrm{~A}$ & 120.0 \\
\hline $\mathrm{C} 1-\mathrm{C} 2-\mathrm{H} 2 \mathrm{~A}$ & 108.7 & $\mathrm{C} 15-\mathrm{C} 14-\mathrm{H} 14 \mathrm{~A}$ & 120.0 \\
\hline $\mathrm{S} 1-\mathrm{C} 2-\mathrm{H} 2 \mathrm{~A}$ & 108.7 & $\mathrm{C} 16-\mathrm{C} 15-\mathrm{C} 14$ & 120.0 \\
\hline N3-C3-N2 & $123.6(4)$ & $\mathrm{C} 16-\mathrm{C} 15-\mathrm{H} 15 \mathrm{~A}$ & 120.0 \\
\hline $\mathrm{N} 3-\mathrm{C} 3-\mathrm{S} 1$ & $122.6(3)$ & $\mathrm{C} 14-\mathrm{C} 15-\mathrm{H} 15 \mathrm{~A}$ & 120.0 \\
\hline $\mathrm{N} 2-\mathrm{C} 3-\mathrm{S} 1$ & $113.8(3)$ & $\mathrm{C} 15-\mathrm{C} 16-\mathrm{C} 11$ & 120.0 \\
\hline $\mathrm{N} 1-\mathrm{C} 4-\mathrm{C} 5$ & $118.6(4)$ & $\mathrm{C} 15-\mathrm{C} 16-\mathrm{H} 16 \mathrm{~A}$ & 120.0 \\
\hline
\end{tabular}




\begin{tabular}{|c|c|}
\hline $\mathrm{N} 1-\mathrm{C} 4-\mathrm{H} 4 \mathrm{~A}$ & 120.7 \\
\hline $\mathrm{C} 5-\mathrm{C} 4-\mathrm{H} 4 \mathrm{~A}$ & 120.7 \\
\hline $\mathrm{C} 10-\mathrm{C} 5-\mathrm{C} 6$ & $118.5(4)$ \\
\hline $\mathrm{C} 10-\mathrm{C} 5-\mathrm{C} 4$ & $120.6(4)$ \\
\hline $\mathrm{C} 6-\mathrm{C} 5-\mathrm{C} 4$ & $120.8(4)$ \\
\hline $\mathrm{C} 7-\mathrm{C} 6-\mathrm{C} 5$ & $119.7(4)$ \\
\hline $\mathrm{C} 7-\mathrm{C} 6-\mathrm{Cl} 1$ & $119.8(4)$ \\
\hline $\mathrm{C} 5-\mathrm{C} 6-\mathrm{Cl} 1$ & $120.4(3)$ \\
\hline $\mathrm{C} 8-\mathrm{C} 7-\mathrm{C} 6$ & $120.7(4)$ \\
\hline $\mathrm{C} 8-\mathrm{C} 7-\mathrm{Cl} 2$ & $119.2(4)$ \\
\hline $\mathrm{C} 6-\mathrm{C} 7-\mathrm{Cl} 2$ & $120.1(4)$ \\
\hline $\mathrm{C} 7-\mathrm{C} 8-\mathrm{C} 9$ & $119.5(5)$ \\
\hline $\mathrm{C} 7-\mathrm{C} 8-\mathrm{H} 8 \mathrm{~A}$ & 120.2 \\
\hline $\mathrm{C} 9-\mathrm{C} 8-\mathrm{H} 8 \mathrm{~A}$ & 120.2 \\
\hline $\mathrm{C} 10-\mathrm{C} 9-\mathrm{C} 8$ & $120.6(5)$ \\
\hline $\mathrm{C} 10-\mathrm{C} 9-\mathrm{H} 9 \mathrm{~A}$ & 119.7 \\
\hline $\mathrm{C} 4-\mathrm{N} 1-\mathrm{N} 2-\mathrm{C} 3$ & $-178.7(4)$ \\
\hline $\mathrm{C} 4-\mathrm{N} 1-\mathrm{N} 2-\mathrm{C} 1$ & $4.3(6)$ \\
\hline $\mathrm{C} 3-\mathrm{N} 2-\mathrm{C} 1-\mathrm{C} 2$ & $16.1(6)$ \\
\hline $\mathrm{N} 1-\mathrm{N} 2-\mathrm{C} 1-\mathrm{C} 2$ & $-166.9(4)$ \\
\hline $\mathrm{N} 2-\mathrm{C} 1-\mathrm{C} 2-\mathrm{C} 11$ & $-141.6(4)$ \\
\hline $\mathrm{N} 2-\mathrm{C} 1-\mathrm{C} 2-\mathrm{S} 1$ & $-19.8(4)$ \\
\hline $\mathrm{C} 3-\mathrm{S} 1-\mathrm{C} 2-\mathrm{C} 11$ & $140.2(3)$ \\
\hline $\mathrm{C} 3-\mathrm{S} 1-\mathrm{C} 2-\mathrm{C} 1$ & $15.9(4)$ \\
\hline $\mathrm{N} 1-\mathrm{N} 2-\mathrm{C} 3-\mathrm{N} 3$ & $-2.2(7)$ \\
\hline $\mathrm{C} 1-\mathrm{N} 2-\mathrm{C} 3-\mathrm{N} 3$ & $175.2(5)$ \\
\hline $\mathrm{N} 1-\mathrm{N} 2-\mathrm{C} 3-\mathrm{S} 1$ & $178.8(3)$ \\
\hline $\mathrm{C} 1-\mathrm{N} 2-\mathrm{C} 3-\mathrm{S} 1$ & $-3.8(5)$ \\
\hline $\mathrm{C} 2-\mathrm{S} 1-\mathrm{C} 3-\mathrm{N} 3$ & $173.2(4)$ \\
\hline $\mathrm{C} 2-\mathrm{S} 1-\mathrm{C} 3-\mathrm{N} 2$ & $-7.8(4)$ \\
\hline $\mathrm{N} 2-\mathrm{N} 1-\mathrm{C} 4-\mathrm{C} 5$ & $175.1(4)$ \\
\hline $\mathrm{N} 1-\mathrm{C} 4-\mathrm{C} 5-\mathrm{C} 10$ & $7.4(6)$ \\
\hline $\mathrm{N} 1-\mathrm{C} 4-\mathrm{C} 5-\mathrm{C} 6$ & $-169.2(4)$ \\
\hline $\mathrm{C} 10-\mathrm{C} 5-\mathrm{C} 6-\mathrm{C} 7$ & $-3.5(6)$ \\
\hline $\mathrm{C} 4-\mathrm{C} 5-\mathrm{C} 6-\mathrm{C} 7$ & $173.1(4)$ \\
\hline $\mathrm{C} 10-\mathrm{C} 5-\mathrm{C} 6-\mathrm{Cl} 1$ & $176.6(3)$ \\
\hline $\mathrm{C} 4-\mathrm{C} 5-\mathrm{C} 6-\mathrm{Cl} 1$ & $-6.8(6)$ \\
\hline $\mathrm{C} 5-\mathrm{C} 6-\mathrm{C} 7-\mathrm{C} 8$ & $2.2(7)$ \\
\hline $\mathrm{C} 11-\mathrm{C} 6-\mathrm{C} 7-\mathrm{C} 8$ & $-177.9(4)$ \\
\hline $\mathrm{C} 5-\mathrm{C} 6-\mathrm{C} 7-\mathrm{Cl} 2$ & $-178.4(3)$ \\
\hline $\mathrm{C} 11-\mathrm{C} 6-\mathrm{C} 7-\mathrm{Cl} 2$ & $1.6(6)$ \\
\hline $\mathrm{C} 6-\mathrm{C} 7-\mathrm{C} 8-\mathrm{C} 9$ & $0.6(8)$ \\
\hline $\mathrm{Cl} 2-\mathrm{C} 7-\mathrm{C} 8-\mathrm{C} 9$ & $-178.8(4)$ \\
\hline $\mathrm{C} 7-\mathrm{C} 8-\mathrm{C} 9-\mathrm{C} 10$ & $-2.1(8)$ \\
\hline
\end{tabular}

$\begin{array}{ll}\mathrm{C} 11-\mathrm{C} 16-\mathrm{H} 16 \mathrm{~A} & 120.0 \\ \mathrm{C} 11-\mathrm{C} 12^{\prime}-\mathrm{C} 13^{\prime} & 123.5(9) \\ \mathrm{C} 11-\mathrm{C} 12^{\prime}-\mathrm{H} 12 \mathrm{~B} & 118.3 \\ \mathrm{C} 13^{\prime}-\mathrm{C} 12^{\prime}-\mathrm{H} 12 \mathrm{~B} & 118.3 \\ \mathrm{C} 14^{\prime}-\mathrm{C} 13^{\prime}-\mathrm{C} 12^{\prime} & 117.0(10) \\ \mathrm{C} 14^{\prime}-\mathrm{C} 13^{\prime}-\mathrm{H} 13 \mathrm{~B} & 121.5 \\ \mathrm{C} 12^{\prime}-\mathrm{C} 13^{\prime}-\mathrm{H} 13 \mathrm{~B} & 121.5 \\ \mathrm{C} 15^{\prime}-\mathrm{C} 14^{\prime}-\mathrm{C} 13^{\prime} & 121.8(10) \\ \mathrm{C} 15^{\prime}-\mathrm{C} 14^{\prime}-\mathrm{H} 14 \mathrm{~B} & 119.1 \\ \mathrm{C} 13^{\prime}-\mathrm{C} 14^{\prime}-\mathrm{H} 14 \mathrm{~B} & 119.1 \\ \mathrm{C} 14^{\prime}-\mathrm{C} 15^{\prime}-\mathrm{C} 16^{\prime} & 118.7(10) \\ \mathrm{C} 14^{\prime}-\mathrm{C} 15^{\prime}-\mathrm{H} 15 \mathrm{~B} & 120.6 \\ \mathrm{C} 16^{\prime}-\mathrm{C} 15^{\prime}-\mathrm{H} 15 \mathrm{~B} & 120.6 \\ \mathrm{C} 11-\mathrm{C} 16^{\prime}-\mathrm{C} 15^{\prime} & 121.7(8) \\ \mathrm{C} 11-\mathrm{C} 16^{\prime}-\mathrm{H} 16 \mathrm{~B} & 119.2\end{array}$

C11-C16'-H16B $\quad 119.2$

C15'-C16'-H16B $\quad 119.2$

$\mathrm{C} 8-\mathrm{C} 9-\mathrm{C} 10-\mathrm{C} 5 \quad 0.7(8)$

$\mathrm{C} 6-\mathrm{C} 5-\mathrm{C} 10-\mathrm{C} 9 \quad 2.1(7)$

$\mathrm{C} 4-\mathrm{C} 5-\mathrm{C} 10-\mathrm{C} 9 \quad-174.5$ (4)

$\mathrm{C} 1-\mathrm{C} 2-\mathrm{C} 11-\mathrm{C} 12 \quad-112.2(5)$

$\mathrm{S} 1-\mathrm{C} 2-\mathrm{C} 11-\mathrm{C} 12$

$\mathrm{C} 1-\mathrm{C} 2-\mathrm{C} 11-\mathrm{C} 16 \quad 69.0(5)$

$\mathrm{S} 1-\mathrm{C} 2-\mathrm{C} 11-\mathrm{C} 16 \quad-50.4$ (4)

$\mathrm{C} 1-\mathrm{C} 2-\mathrm{C} 11-\mathrm{C}_{16} \quad 58.9(10)$

$\mathrm{S} 1-\mathrm{C} 2-\mathrm{C} 11-\mathrm{C} 16^{\prime} \quad-60.6(9)$

$\mathrm{C} 1-\mathrm{C} 2-\mathrm{C} 11-\mathrm{C} 12^{\prime} \quad-117.4(7)$

$\mathrm{S} 1-\mathrm{C} 2-\mathrm{C} 11-\mathrm{C} 12^{\prime} \quad 123.1$ (7)

$\mathrm{C} 16-\mathrm{C} 11-\mathrm{C} 12-\mathrm{C} 13 \quad 0.0$

$\mathrm{C} 2-\mathrm{C} 11-\mathrm{C} 12-\mathrm{C} 13 \quad-178.7(5)$

$\mathrm{C} 11-\mathrm{C} 12-\mathrm{C} 13-\mathrm{C} 14 \quad 0.0$

$\mathrm{C} 12-\mathrm{C} 13-\mathrm{C} 14-\mathrm{C} 15 \quad 0.0$

$\mathrm{C} 13-\mathrm{C} 14-\mathrm{C} 15-\mathrm{C} 16 \quad 0.0$

$\mathrm{C} 14-\mathrm{C} 15-\mathrm{C} 16-\mathrm{C} 11 \quad 0.0$

$\mathrm{C} 12-\mathrm{C} 11-\mathrm{C} 16-\mathrm{C} 15 \quad 0.0$

$\mathrm{C} 2-\mathrm{C} 11-\mathrm{C} 16-\mathrm{C} 15 \quad 178.9$ (4)

$\mathrm{C} 16^{\prime}-\mathrm{C} 11-\mathrm{C} 12^{\prime}-\mathrm{C}_{13}{ }^{\prime} \quad-1.5(15)$

$\mathrm{C} 2-\mathrm{C} 11-\mathrm{C} 12^{\prime}-\mathrm{C} 13^{\prime} \quad 175.4(9)$

$\mathrm{C} 11-\mathrm{C} 12^{\prime}-\mathrm{C} 13^{\prime}-\mathrm{C} 14^{\prime} \quad 1.2(16)$

$\mathrm{C} 12^{\prime}-\mathrm{C} 13^{\prime}-\mathrm{C} 14^{\prime}-\mathrm{C} 15^{\prime} \quad 2.0(16)$

$\mathrm{C} 13^{\prime}-\mathrm{C} 14^{\prime}-\mathrm{C} 15^{\prime}-\mathrm{C} 16^{\prime} \quad-4.9$ (16)

$\mathrm{C} 12^{\prime}-\mathrm{C} 11-\mathrm{C} 16^{\prime}-\mathrm{C} 15^{\prime} \quad-1.5(15)$

$\mathrm{C} 2-\mathrm{C} 11-\mathrm{C} 16^{\prime}-\mathrm{C} 15^{\prime} \quad-177.6(7)$

$\mathrm{C} 14^{\prime}-\mathrm{C} 15^{\prime}-\mathrm{C} 16^{\prime}-\mathrm{C} 11 \quad 4.6(16)$ 
Hydrogen-bond geometry $\left(A,{ }^{\circ}\right)$

$\mathrm{Cg} 3$ and $\mathrm{Cg} 4$ are the centroids of the major (C11-C16) and minor (C11/C12'-C16') disorder components, respectively, of the phenyl ring.

\begin{tabular}{|c|c|c|c|c|}
\hline$D-\mathrm{H} \cdots A$ & $D-\mathrm{H}$ & $\mathrm{H} \cdots A$ & $D \cdots A$ & $D-\mathrm{H} \cdots A$ \\
\hline $\mathrm{N} 3-\mathrm{H} 3 A \cdots \mathrm{Br} 1^{\mathrm{i}}$ & 0.90 & 2.51 & $3.303(4)$ & 147 \\
\hline $\mathrm{N} 3-\mathrm{H} 3 B \cdots \mathrm{Br} 1$ & 0.90 & 2.36 & $3.258(4)$ & 175 \\
\hline $\mathrm{C} 13^{\prime}-\mathrm{H} 13 B^{\cdots} C^{\prime} 3^{\mathrm{ii}}$ & 0.93 & 2.91 & $3.596(12)$ & 132 \\
\hline $\mathrm{C} 13^{\prime}-\mathrm{H} 13 B^{\cdots}{ }^{\prime} C g 4^{\mathrm{ii}}$ & 0.93 & 2.99 & $3.746(12)$ & 139 \\
\hline $\mathrm{C} 2-\mathrm{H} 2 A \cdots \mathrm{Br} 1^{\mathrm{iii}}$ & 0.98 & 2.87 & $3.778(5)$ & 154 \\
\hline $\mathrm{C} 10-\mathrm{H} 10 A \cdots \mathrm{Br} 1^{\mathrm{i}}$ & 0.93 & 2.90 & $3.796(5)$ & 161 \\
\hline $\mathrm{C} 7-\mathrm{Cl} 2 \cdots C g 3^{\text {iv }}$ & $1.73(1)$ & $3.80(1)$ & $5.525(6)$ & $175(1)$ \\
\hline $\mathrm{C} 7-\mathrm{Cl} 2 \cdots C g 4^{\mathrm{iv}}$ & $1.73(1)$ & $3.57(1)$ & $5.299(6)$ & $175(1)$ \\
\hline
\end{tabular}

Symmetry codes: (i) $-x+1,-y+2,-z+1$; (ii) $-x+1, y-1 / 2,-z+3 / 2$; (iii) $x, y-1, z$; (iv) $-x+2,-y,-z+1$. 